\title{
A Variational Framework for Joint Detection and Segmentation of Ovarian Cancer Metastases
}

\author{
Jianfei Liu ${ }^{1}$, Shijun Wang ${ }^{1}$, Marius George Linguraru ${ }^{2}$, Jianhua Yao ${ }^{1}$, \\ and Ronald M. Summers ${ }^{1}$ \\ 1 Imaging Biomarkers and Computer-Aided Diagnosis Laboratory, \\ Radiology and Imaging Science, National Institutes of Health Clincial Center, \\ Bethesda, MD 20892 \\ 2 Sheikh Zayed Institute for Pediatric Surgical Innovation, \\ Childrens National Medical Center, Washington, DC 20010
}

\begin{abstract}
Detection and segmentation of ovarian cancer metastases have great clinical impacts on women's health. However, the random distribution and weak boundaries of metastases significantly complicate this task. This paper presents a variational framework that combines region competition based level set propagation and image matching flow computation to jointly detect and segment metastases. Image matching flow not only detects metastases, but also creates shape priors to reduce over-segmentation. Accordingly, accurate segmentation helps to improve the detection accuracy by separating flow computation in metastasis and non-metastasis regions. Since all components in the image processing pipeline benefit from each other, our joint framework can achieve accurate metastasis detection and segmentation. Validation on 50 patient datasets demonstrated that our joint approach was superior to a sequential method with sensitivity $89.2 \%$ vs. $81.4 \%$ (Fisher exact test $p=0.046$ ) and false positive per patient 1.04 vs. 2.04. The Dice coefficient of metastasis segmentation was $92 \pm 5.2 \%$ vs. $72 \pm 8 \%$ (paired t-test $p=0.022$ ), and the average surface distance was $1.9 \pm 1.5 \mathrm{~mm}$ vs. $4.5 \pm 2.2 \mathrm{~mm}$ (paired t-test $p=0.004)$.
\end{abstract}

Keywords: Ovarian Cancer Metastasis, Joint Detection and Segmentation, Level Set, Dynamic Shape Prior.

\section{Introduction}

Detection and segmentation of ovarian cancer metastases contribute to the prognosis and treatment of women with ovarian cancer because $75 \%$ of them already have metastases spreading to the upper abdomen[5]. This task suffers many challenges. Metastases randomly touch organs in Fig. 1a they have weak boundaries with surrounding fluids in Fig. 1b: they also present wide ranges of shapes, spherical structures in Fig. 1a and elongated shapes in Fig. 1c. Because of these challenges, there is very limited work on this important topic. We developed a tumor sensitive matching flow to detect metastases [3]. However, this method yields metastasis over-segmentation caused by weak metastasis boundaries.

K. Mori et al. (Eds.): MICCAI 2013, Part II, LNCS 8150, pp. 83-90 2013.

(C) US Government (outside the US) 2013 


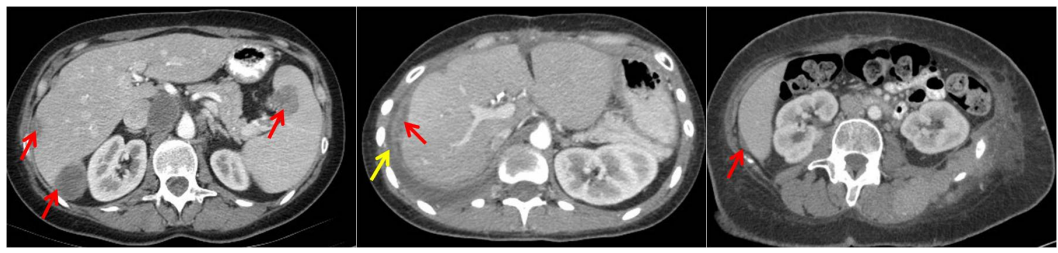

(a) Random distribution

(b) Weak boundary

(c) Varying shape

Fig. 1. Challenges in detecting and segmenting ovarian cancer metastases, where red arrows indicate metastases and the yellow arrow points to fluids

Joint detection and segmentation is a potential solution because metastasis detection indicates tumor locations while segmentation improves the accuracy of feature descriptors to detect tumors. Due to benefits from both sides, joint detection and segmentation of objects receive high research interests. Conditional random field (CRF) 2] was often exploited because CRF can predict a label for sequences of input samples. Singaraju[7] introduced bag of features as the global feature descriptor and integrated them into CRF. Shotton 6 combined multiple visual cues to detect objects. In contrast to ovarian cancer metastasis, the objects detected in these methods have clear boundaries. Yezzi 9 developed a variational framework to evolve active contours to simultaneously carry out segmentation and registration on brain images. Joint segmentation and registration on MRI images was also studied in Wyatt's work [8, except that Markov random field was chosen to establish the framework. Lu[4] equipped Wyatt's framework with the capability of tumor detection by introducing tumor shape priors. However, varying metastasis shapes hinder this method to deal with our problems.

In this paper, we propose a novel joint detection and segmentation approach by integrating level set propagation and image matching flow computation within a variational framework. The main contribution is three-fold. First, the joint framework performs a tumor sensitive image matching process to steer the matching flow towards the metastasis regions, and metastases are detected by searching for large flow vectors. Second, the joint framework is also a level set segmentation process by using image regions with large matching flow vectors as shape priors to guide metastasis segmentation. Third, metastasis segmentation improves the accuracy of image matching flow by separating its computation in metastasis and non-metastasis regions. The validation on 50 patient datasets demonstrated that our joint framework outperforms the sequential approach 3 ] with more accurate metastasis detection and segmentation.

\section{Variational Framework}

This section describes a variational framework for joint detection and segmentation of ovarian cancer metastases. The framework not only integrates tumor sensitive matching flow computation 3] and region competition based level set 
segmentation [1, but also allow them to benefit from each other to yield accurate metastasis detection and segmentation.

One rule of our joint framework is to embed a metastasis-likelihood function $C(\mathbf{p})$ into the image matching process to identify metastasis-caused deformation in the abdomen, where $\mathbf{p}=(x, y, z) . C(\mathbf{p})$ is defined in 3 based on the assumptions that the organ surface is locally presented as a cup shape and metastasis intensity values obey mixture Gaussian model trained from six patient datasets. Image matching flow $\mathbf{u}(\mathbf{p})=\left(u_{x}(\mathbf{p}), u_{y}(\mathbf{p}), u_{z}(\mathbf{p})\right)$ is computed by comparing the patient image $I_{p}(\mathbf{p})$ (Fig. 2a $)$ and the reference image $I_{r}(\mathbf{p})$ (Fig. 2b) as well as distance maps $D_{p}(\mathbf{p})$ (Fig. 2c) and $D_{r}(\mathbf{p})$ (Fig. 2d) representing the shapes of the patient organ and the probabilistic atlas. Here, $I_{r}(\mathbf{p})$ is created from a random CT image of a healthy subject. Additionally, we seek two sets of level set functions $\psi_{i}(\mathbf{p}), i=1,2$, that partitions the patient image domain $\Omega$ into metastasis regions $\Omega_{1}$ and non-metastasis regions $\Omega_{2}$. To allow image matching to benefit from image segmentation, we estimate two flow fields $\mathbf{u}_{1}$ and $\mathbf{u}_{2}$ for metastasis and non-metastasis regions. Our joint framework is thus formulated by integrating image matching and segmentation into a variational functional.

$$
\begin{aligned}
& E(\mathbf{u}, \psi)=\sum_{i=1}^{2} \int_{\Omega} H\left(\psi_{i}\right)\left(\Psi(\underbrace{\left|I_{r}\left(\mathbf{p}+\mathbf{u}_{i}\right)-I_{p}(\mathbf{p})\right|^{2}}_{\text {Intensity Constancy }})+\alpha C(\mathbf{p}) \Psi(\underbrace{\left|\nabla I_{r}\left(\mathbf{p}+\mathbf{u}_{i}\right)-\nabla I_{p}(\mathbf{p})\right|^{2}}_{\text {Gradient Constancy }})\right. \\
& \left.+\beta C(\mathbf{p}) \Psi(\underbrace{\left|D_{r}\left(\mathbf{p}+\mathbf{u}_{i}\right)-D_{p}(\mathbf{p})\right|^{2}}_{\text {Distance Constancy }})\right) d \mathbf{p}-\sum_{i=1}^{2} \int_{\Omega} H\left(\psi_{i}\right)(\underbrace{v \log P_{i}\left(I_{p}(\mathbf{p})\right)}_{\text {Image Term }}+\eta(\underbrace{\log P_{i}\left(\left\|\mathbf{u}_{i}\right\|\right)}_{\text {Flow Term }})) d \mathbf{p} \\
& +\sum_{i=1}^{2} \int_{\Omega}(\gamma \Psi(\underbrace{\left|\nabla\left(u_{x}\right)_{i}\right|^{2}+\left|\nabla\left(u_{y}\right)_{i}\right|^{2}+\left|\nabla\left(u_{z}\right)_{i}\right|^{2}}_{\text {Flow Smoothness }})+\kappa \underbrace{\left|\nabla H\left(\psi_{i}\right)\right|}_{\text {Contour Area }}) d \mathbf{p}
\end{aligned}
$$

where $H\left(\psi_{i}\right)$ is a Heaviside function[1] and $\Psi\left(s^{2}\right)=\sqrt{s^{2}+0.001^{2}}$ is a modified L1 norm that allows for handling outliers. $\alpha=550, \beta=1000, \kappa=1.5$, and $\gamma=5500$, where are given by Brox 1 . $\eta=2000$ and $v=1$ are experimentally determined. A statistical model is used to compose the image term and the flow term, which is given by $P_{i}(s)=\frac{1}{\sqrt{2 \pi} \sigma_{i}} \exp \left(-\frac{\left(s-\mu_{i}\right)^{2}}{2 \sigma_{i}^{2}}\right)$ with the mean value $\mu_{i}$ and standard deviation $\sigma_{i}$ in region $i$.

Image matching flow is computed by intensity, gradient and distance constancy models as well as the flow smoothness assumption in Eq. 1. Adjusting the weights of gradient and distance constancy terms by $C(\mathbf{p})$ can force flow vectors only to be magnified in the metastases regions. Fig. 2e illustrates a metastasis touching the liver, and Fig. 2f] shows that this metastasis can be detected by searching for image matching flow vectors with their lengths larger than $10 \mathrm{~mm}$ as clinically relevant. Different from [3], our approach separates the matching flow computation in metastasis and non-metastasis regions by $H\left(\psi_{i}\right)$. This partition strategy can leverage the accuracy of metastasis detection by $C(\mathbf{p})$.

Eq. 1 can also be treated as a region competition based segmentation scheme 1] where the data term includes statistical models of intensity and flow magnitude 


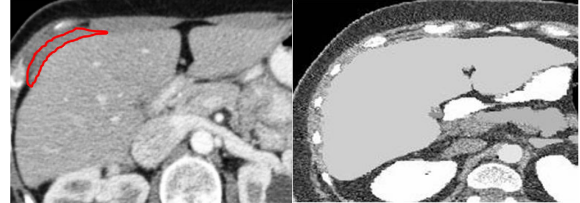

(a) (b)

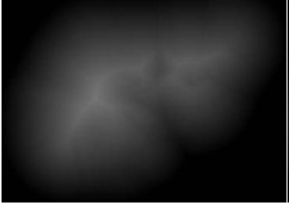

(c)

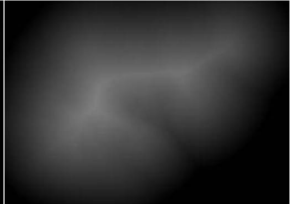

(d)

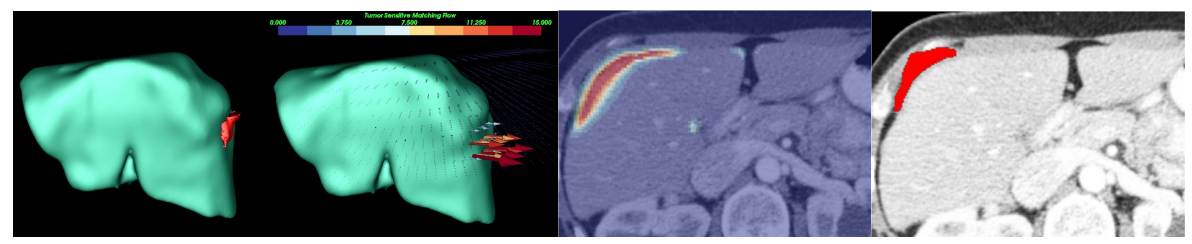

(e)

(f)

$(\mathrm{g})$

(h)

Fig. 2. Our joint framework for metastasis detection and segmentation. (a) A patient image with metastases (inside red contours) attaching to liver, (b) reference image, (c) distance map of the segmented liver, (d) distance map of the atlas, (e) metastases in $3 \mathrm{D}$, (f) image matching flow, (g) shape prior constructed by image regions in red with large flow vectors, (h) metastasis segmentation.

in the second integral as well as the tumor sensitive matching flow computation in the first integral. We can note that the metastasis is roughly represented by image regions in red with large flow vectors in Fig. $2 \mathrm{~g}$. This is a desirable property to address the over-segmentation issue, as it dynamically provides shape priors adaptable to the current patient. The level set driven by the flow vector competition will be balanced approximately at metastasis boundaries. Competing intensity statistical model at metastasis and non-metastasis regions can refine the level set propagation constrained by the shape prior and ensure the level sets to stop at the metastasis boundaries. In addition, the area of the region contours is also penalized by $\kappa$ in the third integral. Fig. $2 \mathrm{~h}$ gives the final metastasis segmentation, which is similar to the metastasis groundtruth in Fig. 2a.

Therefore, minimizing Eq. 1 1 with respect to $\mathbf{u}$ and $\psi$ can jointly detect (Fig. 2ff) and segment (Fig. 2h) ovarian cancer metastases, and the final metastasis segmentation is inside the contour $\psi_{1}$.

\section{Optimization}

Eq. 1 is non-trivial to solve because it is a non-linear and non-convex energy functional, which might have several local optima. Multi-scale image representation [13] is an efficient means to deal with this issue as multiple optima are rare in the coarse scale. 


\subsection{Initialization of the Joint Framework at the Coarsest Scale}

The robust initialization of unknown variables $\mathbf{u}$ and $\psi$ in Eq. 1 is critical to avoid local optimal as well as improve the accuracy of the metastasis detection by $C(\mathbf{p})$. In this work, $\mathbf{u}$ is initialized by performing tumor sensitive matching flow computation 3 at coarse scale.

$\psi$ can be initialized by segmenting the matching flow $\mathbf{u}$. Since metastasis and non-metastasis regions are distinguished in terms of the vector magnitudes, we can employ the two-region segmentation strategy described in [1] to perform the flow segmentation.

$$
E(\psi)=\int_{\Omega}\left(-H(\psi) \log P_{1}(\|\mathbf{u}\|)-(1-H(\psi)) \log P_{2}(\|\mathbf{u}\|)+\kappa|\nabla H(\psi)|\right) d \mathbf{p}
$$

Here, $P(\|\mathbf{u}\|)$ follows the definition in Eq. 1. Finally, $\psi$ in Eq. 1] can be assigned by the results of Eq. 2 .

\subsection{Minimization}

Let's first define some abbreviations to simplify the description.

$$
\begin{aligned}
& \left(I_{x}\right)_{i}=\partial_{x} I_{r}\left(\mathbf{p}+\mathbf{u}_{i}\right) \quad\left(I_{x x}\right)_{i}=\partial_{x x} I_{r}\left(\mathbf{p}+\mathbf{u}_{i}\right) \quad(\Delta I)_{i}=I_{r}\left(\mathbf{p}+\mathbf{u}_{i}\right)-I_{p}(\mathbf{p}) \\
& \left(I_{x y}\right)_{i}=\partial_{x x} I_{r}\left(\mathbf{p}+\mathbf{u}_{i}\right) \quad\left(I_{x z}\right)_{i}=\partial_{x x} I_{r}\left(\mathbf{p}+\mathbf{u}_{i}\right) \quad\left(\Delta_{x} I\right)_{i}=\partial_{x} I_{r}\left(\mathbf{p}+\mathbf{u}_{i}\right)-\partial_{x} I_{p}(\mathbf{p})
\end{aligned}
$$

The abbreviations for $\mathrm{y}$ and $\mathrm{z}$ components and distance map $D$ can be similarly derived according to the rules defined in Eq. 3. The Euler-Lagrange equation to minimize $\left(u_{x}\right)_{i}$ in Eq. 1 is given by

$$
\begin{aligned}
H\left(\psi_{i}\right) & \left(\Psi^{\prime}\left((\Delta I)_{i}^{2}\right)\left(I_{x}\right)_{i}(\Delta I)_{i}+\alpha C(\mathbf{p}) \Psi^{\prime}\left(\left(\Delta_{x} I\right)_{i}^{2}+\left(\Delta_{y} I\right)^{2}+\left(\Delta_{z} I\right)_{i}^{2}\right)\left(\left(I_{x x}\right)_{i}\left(\Delta_{x} I\right)_{i}\right.\right. \\
& \left.\left.+\left(I_{x y}\right)_{i}\left(\Delta_{y} I\right)_{i}+\left(I_{x z}\right)_{i}\left(\Delta_{z} I\right)_{i}\right)+\beta C(\mathbf{p}) \Psi^{\prime}\left((\Delta D)_{i}^{2}\right)\left(D_{x}\right)_{i}(\Delta D)_{i}\right) \\
& -\gamma \operatorname{div}\left(\Psi^{\prime}\left(\left|\nabla\left(u_{x}\right)_{i}\right|^{2}+\left|\nabla\left(u_{y}\right)_{i}\right|^{2}+\left|\nabla\left(u_{z}\right)_{i}\right|^{2}\right) \nabla\left(u_{x}\right)_{i}\right)=0
\end{aligned}
$$

Similar equations can be derived for $\left(u_{y}\right)_{i}$ and $\left(u_{z}\right)_{i}$ components. The nested iteration approach[1] is applied to remove non-linearity and non-convexity in Eq. 4. Note that image matching flow $\mathbf{u}_{i}$ is computed only within the image regions confined by the Heaviside function $H\left(\psi_{i}\right)$. This is a beneficial attribute because non-metastasis regions cannot affect image matching flow computation in the metastasis regions, which yields more accurate matching flow to detect metastases in comparison with [3].

The Euler-Lagrange equation regarding to $\psi_{i}$ is formulated as

$$
\begin{gathered}
H^{\prime}\left(\psi_{i}\right)\left(-\Psi\left((\Delta I)_{i}^{2}\right)-\alpha C(\mathbf{p}) \Psi\left(\left(\Delta_{x} I\right)_{i}^{2}+\left(\Delta_{y} I\right)^{2}+\left(\Delta_{z} I\right)_{i}^{2}\right)-\beta C(\mathbf{p}) \Psi\left((\Delta D)_{i}^{2}\right)\right. \\
\left.+v \log P_{i}\left(I_{p}(\mathbf{p})\right)+\eta \log P_{i}\left(\left\|\mathbf{u}_{\mathbf{i}}\right\|\right)+\kappa \operatorname{div}\left(\frac{\nabla \psi_{i}}{\left|\nabla \psi_{i}\right|}\right)\right)=0
\end{gathered}
$$


We employ the region competition strategy developed in [1] to derive a gradient descent form of level set propagation.

$$
\begin{aligned}
& \partial_{t} \psi_{i}=H^{\prime}\left(\psi_{i}\right)\left(e_{i}-\max _{\substack{H^{\prime}\left(\psi_{j}\right)>0.3 \\
j \neq i}}\left(e_{j}, e_{i}-1\right)\right) \\
& e_{i}=-\left(\Psi\left((\Delta I)_{i}^{2}\right)+\alpha C(\mathbf{p}) \Psi\left(\left(\Delta_{x} I\right)_{i}^{2}+\left(\Delta_{y} I\right)^{2}+\left(\Delta_{z} I\right)_{i}^{2}\right)+\beta C(\mathbf{p}) \Psi\left((\Delta D)_{i}^{2}\right)\right) \\
& /\left(\frac{1}{|\Omega|} \int_{\Omega}\left(\Psi\left((\Delta I)_{i}^{2}\right)+\alpha C(\mathbf{p}) \Psi\left(\left(\Delta_{x} I\right)_{i}^{2}+\left(\Delta_{y} I\right)^{2}+\left(\Delta_{z} I\right)_{i}^{2}\right)+\beta C(\mathbf{p}) \Psi\left((\Delta D)_{i}^{2}\right)\right) d \mathbf{p}\right) \\
& +v \log P_{i}\left(I_{p}(\mathbf{p})\right)+\eta \log P_{i}\left(\left\|\mathbf{u}_{\mathbf{i}}\right\|\right)+\kappa \operatorname{div}\left(\frac{\nabla \psi_{i}}{\left|\nabla \psi_{i}\right|}\right)
\end{aligned}
$$

Here, $\psi_{i}$ is propagated by metastasis $\left(e_{i}\right)$ and non-metastasis $\left(e_{j}\right)$ region competition according to image matching flow computation, statistical models of intensity and flow magnitude, and the curvature term restricted to the narrow band given by $H^{\prime}\left(\psi_{i}\right)>0.3$. As discussed before, the image matching flow competition creates a dynamic shape prior because image regions with large flow vectors roughly represent the metastasis shapes. The competition between intensity models contributes to achieving accurate metastasis segmentation.

\section{Experimental Data and Results}

Our joint framework was validated on abdominal contrast-enhanced CT images from 50 patients (excluding six patients used for $C(\mathbf{p})$ in Eq. 1). Slice thickness varies from $1.0 \mathrm{~mm}$ to $2.0 \mathrm{~mm}$. Retrospective analysis of all images was approved by our IRB. 25 patients had at least one ovarian cancer metastasis. 102 metastases in these images were annotated by an experienced radiologist and used as the ground-truth. 92 metastases were attached to the liver, and the remaining 10 to the spleen. The size range of all metastases was $2.3-52.3 \mathrm{~mm}$ (average: $21.1 \pm 12.8 \mathrm{~mm})$.

Table 1. Accuracy evaluation of metastasis detection and segmentation

\begin{tabular}{|c|c|c|c|c|c|c|c|c|}
\hline Method & Sen.(\%) & FP/Pat. & VO(\%) & DC $(\%)$ & RV(\%) & AD $(\mathrm{mm})$ & SD $(\mathrm{mm})$ & MD (mm) \\
\hline Sequential & 81.4 & 2.04 & $57 \pm 10$ & $72 \pm 8$ & $33 \pm 22$ & $4.5 \pm 2.2$ & $7.8 \pm 3.2$ & $42 \pm 9.4$ \\
\hline Joint & 89.2 & 1.04 & $82 \pm 7.1$ & $92 \pm 5.2$ & $14 \pm 10$ & $1.9 \pm 1.5$ & $3.8 \pm 2.4$ & $18 \pm 7.2$ \\
\hline
\end{tabular}

Eight metrics used in [4] were chosen to evaluate the accuracy of metastasis detection and segmentation. Sensitivity (Sen.) and false positive rate per patient (FP/Pat.) were used to evaluate detection results. FP was defined as independently segmented regions with which less than $50 \%$ of expert annotations overlap. The segmentation accuracy was appraised by volume overlap (VO), Dice coefficient (DC), relative absolute volume difference (RV), average symmetric absolute surface distance (AD), symmetric RMS surface distance (SD), and maximum symmetric absolute surface distance (MD). Table11indicates that 


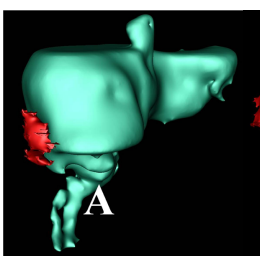

(a)

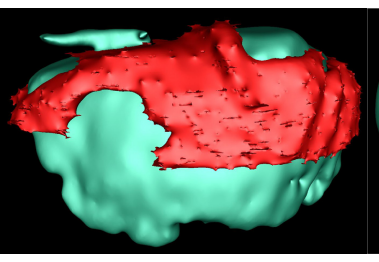

(b)

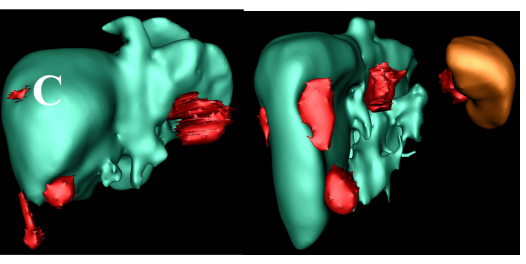

(c) (d)

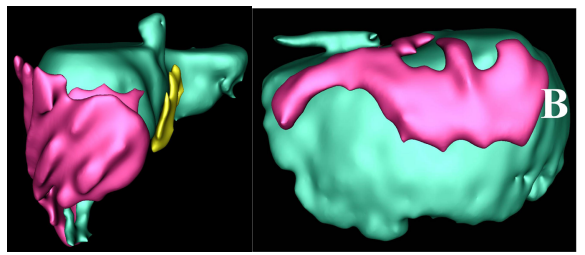

(e) (f)

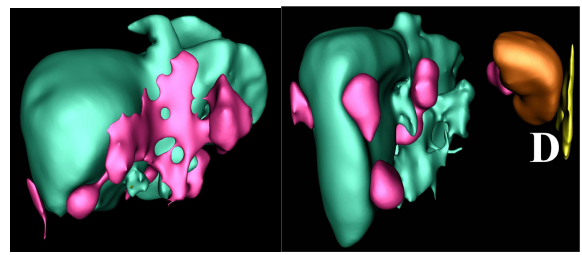

(g) (h)

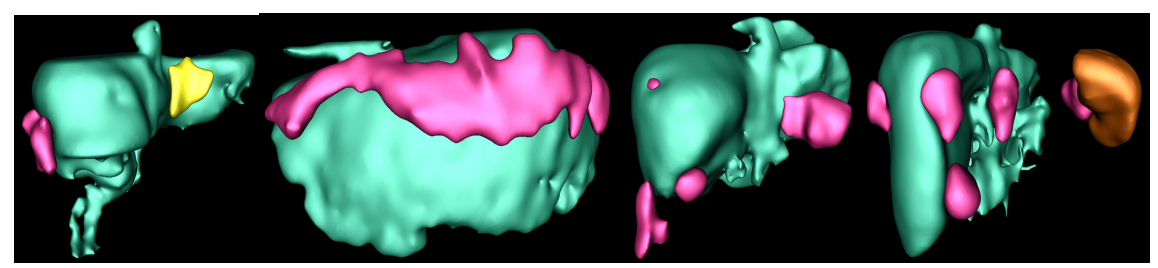

(i)

(j)

$(\mathbf{k})$

(1)

Fig. 3. Comparison of metastasis detection and segmentation using sequential and joint approaches. Top row: ground-truth metastases are in red, liver in green and spleen in brown. Center row: results using sequential approach, where true detections in red and false positives in yellow. Bottom row: joint method.

the sensitivity of the joint framework increased substantially compared to the sequential method 3 (Fisher exact test $p=0.046$ ) with half the number of FP/Pat because metastasis segmentation separates image matching flow computation in metastasis and non-metastasis regions. The segmentation accuracy is also significantly improved due to the dynamical shape prior constructed by the joint framework ( $p=0.004$, paired t-test on DC).

Fig. 3 compares metastasis detection and segmentation using sequential and joint approaches. Each column represents one patient. In Fig. 3a, part of liver is removed in the ' $\mathrm{A}$ ' region and the abdomen also contains fluids. Without shape constraints, the metastasis was significantly over-segmented by the sequential method [3] in Fig. 3e. In contrast, joint approach addressed over-segmentation in Fig. 3i. The second column shows a patient with metastasis clusters warping the liver. Although both sequential and joint methods can detect them, metastases in area 'B' of Fig. [3f are missed by the sequential method while recovered by joint approach. The joint approach can also detect a small metastasis in area 
' $\mathrm{C}$ ' in Fig. 3k while missed by the sequential method. The fourth patient reveals that joint method can reduce false positives, such as one at ' $\mathrm{D}$ ' in Fig. 3h. These four examples support the findings in Table. 1.

\section{Conclusion and Future Work}

In this paper, we developed a variational framework to jointly detect and segment ovarian cancer metastases by unifying image matching flow computation and region competition based level set propagation. The validation results demonstrated that our joint framework generates more accurate metastasis detection and segmentation than sequential method [3]. In the future, we will investigate metastasis texture features to enhance the detection accuracy.

Ackowledgement. This work was supported by the Intramural Research Program of the National Institutes of Health, Clinical Center.

\section{References}

1. Brox, T., Bruhn, A., Weickert, J.: Variational motion segmentation with level sets. In: Leonardis, A., Bischof, H., Pinz, A. (eds.) ECCV 2006, Part I. LNCS, vol. 3951, pp. 471-483. Springer, Heidelberg (2006)

2. Lafferty, J., McCallum, A., Pereira, F.: Conditional random fields: Probabilistic models for segmenting and labeling sequence data. In: ICML, pp. 282-289 (2001)

3. Liu, J., Wang, S., Linguraru, M.G., Summers, R.M.: Tumor sensitive matching flow: an approach for ovarian cancer metastasis detection and segmentation. In: Yoshida, H., Hawkes, D., Vannier, M.W. (eds.) Abdominal Imaging 2012. LNCS, vol. 7601, pp. 188-197. Springer, Heidelberg (2012)

4. Lu, C., Chelikani, S., Duncan, J.S.: A unified framework for joint segmentation, nonrigid registration and tumor detection: Application to MR-guided radiotherapy. In: Székely, G., Hahn, H.K. (eds.) IPMI 2011. LNCS, vol. 6801, pp. 525-537. Springer, Heidelberg (2011)

5. Memarzadeh, S., Berek, J.: Advances in the management of epithelial ovarian cancer. J. Reprod. Med. 46(7), 621-629 (2001)

6. Shotton, J., Winn, J., Rother, C., Criminisi, A.: Textonboost for image understanding: Multi-class object recognition and segmentation by jointly modeling texture, layout, and context. Int. J. of Comp. Vis. 81(1), 2-23 (2009)

7. Singaraju, D., Vidal, R.: Using global bag of features models in random fields for joint categorization and segmentation of objects. In: CVPR, pp. 2313-2319 (2011)

8. Wyatt, P., Noble, J.: Map mrf joint segmentation and registration of medical images. Medical Image Analysis 7, 539-552 (2003)

9. Yezzi, A., Zollei, L., Kapur, T.: A variational framework for joint segmentation and registration. In: MMBIA, pp. 44-51 (2001) 